\title{
A unique theory of all forces
}

\author{
Paolo Di Vecchia \\ NORDITA \\ Blegdamsvej 17, DK-2100 Copenhagen Ø, Denmark \\ Talk given at the Conference "Beyond the Standard Model" , V \\ Balholm, Norway (May 1997)
}

\begin{abstract}
In discussing the construction of a consistent theory of quantum gravity unified with the gauge interactions we are naturally led to a string theory. We review its properties and the five consistent supersymmetric string theories in ten dimensions. We finally discuss the evidence that these theories are actually special limits of a unique 11-dimensional theory, called M-theory, and a recent conjecture for its explicit formulation as a supersymmetric Matrix theory.
\end{abstract}

\section{Unification of all interactions}

The electro-weak and strong interactions are described by the so-called standard model of particle physics. It is a gauge field theory based on the direct product of the group $S U(3)$ colour with coupling constant $g_{3}$ for the strong interactions and the group $S U(2) \otimes U(1)$ with coupling constants $g_{2}$ and $g_{1}$ respectively for the electro-weak interactions. A non abelian gauge theory is a generalization of quantum electrodynamics (QED) to the case of a non-abelian gauge group and is described by a Lagrangian of the type:

$$
\mathcal{L}=-\frac{1}{4 g^{2}} F_{\mu \nu}^{2}+\bar{\Psi}\left(i \gamma^{\mu} D_{\mu}-m\right) \Psi \quad ; \quad D_{\mu}=\partial_{\mu}+i A_{\mu}
$$

The field $\Psi$ describes the matter fields that in the standard model are the quarks and leptons, while the gauge field $A_{\mu}$ and its field strenght $F_{\mu \nu}$ describe the gauge particles responsible for providing the interaction among the matter particles. In the standard model the gauge fields are the 8 gluons for the strong interactions and the $W^{ \pm}$, the $Z^{0}$ and the photon for the electro-weak interactions. 
When one quantizes $Q E D$ or the standard model one finds infinities that are the reflection of the infinities that are already present at the classical level. Remember that, for instance, already in classical electromagnetism [1] one must introduce a classical radius $r_{0}$ for the electron, in order to avoid short distances divergences directly related to the pointlike nature of the electron, defined by the $e^{2} / r_{0}=m c^{2}$. These infinities persist in the quantum theory and must be renormalized away in order to obtain finite quantities to be compared with the experiments. One of the most important consequences of the renormalization theory is the fact that the coupling constants are not fixed once and for all as in the classical theory, but are running with the energy at which we perform the experiments. Their running is completely fixed by the particle spectrum at low energy and by their quantum numbers under the gauge group of the standard model, at least up to an energy at which new physics and new particles appear. Since the three gauge coupling constants of the standard model have been measured with very high precision at LEP at an energy equal to the mass of the $Z^{0}$ gauge boson and one knows the particle spectrum of the standard model one can see if they will meet at high energy. It turns out that they do not meet at a common value in the case of the standard model [2], but, if we extend the standard model by introducing for each particle of the standard model a supersymmetric partner obtaining the spectrum of the minimal supersymmetric standard model, then one can choose the supersymmetric breaking scale comparable with the electro-weak scale $\left(10^{2}, 10^{3} \mathrm{GeV}\right)$ in such a way that the three couplings now meet in a single point [2], that we call $\alpha_{G U T}$, at an energy $M_{G U T}$. The values found for these two quantities at the unification point are

$$
M_{G U T}=2 \cdot 10^{16} \mathrm{GeV} \quad \alpha_{G U T} \equiv \frac{g_{G U T}^{2}}{4 \pi} \sim \frac{1}{25}
$$

Supersymmetry is not only consistent with the fact that the three couplings unify at high energy, but it is also required for other reasons as for instance the hierarchy problem. Because of them it is believed, but far from proved, that at an energy of the order of the electro-weak scale the standard model has to be modified to include supersymmetry. Supersymmetry generalizes the Lorentz invariance of special relativity theory and the translational invariance responsible for the energy conservation, in a symmetry where bosons and fermions coexist in the same multiplets.

The previous considerations imply that at sufficiently high energy also the strong interactions will be unified with the electro-weak interactions. This is, of course, a very appealing idea, but at this point we must not forget that in nature we observe another kind of interaction, the gravitational interaction. The gravitational interactions are described by the Einstein's theory of general relativity in which the gravitational force is produced by the fact that the space-time is curved. The action for the gravitational field is given by the Einstein's action:

$$
S=\frac{c^{3}}{16 \pi G_{N}} \int d^{4} x \sqrt{-g} R
$$


where $R$ is the scalar curvature of the space-time and $G_{N}$ is the Newton constant that in the newtonian limit enters in the gravitational force $F$ between two equal masses $M$ at a distance $R$ given by $F=-G_{N} M^{2} / R^{2}$. The gravitational attraction between two masses becomes strong when the dimensionless coupling constant $G_{N} M^{2} / \hbar c \sim 1$. This equality defines the Planck mass given by

$$
M_{P}=\sqrt{\frac{\hbar c}{G_{N}}}=1.2 \cdot 10^{19} \mathrm{GeV}
$$

When we approach energies of the order of the Planck mass then also gravity must be quantized, but Einstein's theory of general relativity is not a renormalizable theory as are gauge theories. This is due to the fact that a gauge field couples to the charge, while gravity couples to the energy giving extra positive powers of the momenta circulating in the loops and making the loop integration more divergent so that the theory becomes non-renormalizable. In other words in the case of gravity the pointlike structure of field theory creates such divergences that the theory cannot be given a quantum meaning at least in perturbation theory.

We face therefore the problem of defining a quantum theory of gravity. Another question has to do with the unification that we have seen between the strong and the electro-weak interactions at a scale that is not so different from the Planck scale. If the strong and the electro-weak interactions are really unified at a unification scale that is only a factor $10^{3}$ smaller than the Planck scale why not to try to unify all interactions at a sufficiently high energy? How this can be done is discussed in the next section.

\section{From point particles to strings}

We have previously stressed that the ultraviolet infinities of the various field theories are due to the fact that they describe pointlike objects. In the case of gravity the short distance divergences are so strong that, if we want to construct a quantum theory of gravity, we are obliged to abandon the idea that the fundamental constituents of nature are pointlike objects. The simplest possible extension is of course that they are tiny one-dimensional strings.

The string model originated at the end of the sixties as an attempt to describe, in the framework of $S$-matrix theory, the physics of strong interactions with an infinite set of resonances, but it became soon clear that it incorporated also massless gauge fields and gravitons that are not present in the low energy hadron spectrum. It predicted also an exponential decrease of the cross sections at large transverse momentum in contradiction with the experiments which showed instead only a power decrease typical of pointlike structures. These unphysical features of string model brought back the research on strong interactions in the realm of field theory and only a few years later the theory of quantum chromodynamics (QCD) was formulated as the one correctly describing the strong interactions. 
On the other hand, exactly for those features that were in disagreement with the physics of strong interactions, it was soon recognized that the string model could provide a theory in which all interactions including gravity were unified [3]. In particular the exponential cutoff at high momentum was an essential feature of the model for providing a finite quantum theory of gravity.

As a spinless point particle is described by its position $x^{\mu}(\tau)$ in Minkowski space-time as a function of its proper time $\tau$, so a bosonic string is described by its position $x^{\mu}(\tau, \sigma)$ as a function of $\tau$ and of an additional variable $\sigma$ that parametrizes the various points of a string. In going from zero-dimensional to one-dimensional objects the free action for a particle becomes that of a string:

$$
S_{p}=-\frac{m c}{2} \int\left(\frac{\partial x}{\partial \tau}\right)^{2} d \tau \Longrightarrow S_{s}=-\frac{T c}{2} \int d \tau \int d \sigma\left[\left(\frac{\partial x}{\partial \tau}\right)^{2}-\left(\frac{\partial x}{\partial \sigma}\right)^{2}\right]
$$

where $m$ is the mass of the particle, while $T$ has the dimension of an energy per unit lenght and it is called the string tension.

If a particle has also non zero spin for instance equal to $1 / 2$ we need to introduce together with its position in the space-time $x^{\mu}(\tau)$ also a Grassmann coordinate $\psi^{\mu}(\tau)$ describing its spin degrees of freedom. In the same way a string having also spin $1 / 2$ degrees of freedom distributed along the string is also described by its Minkowski coordinate $x^{\mu}(\tau, \sigma)$ together with a Grassmann coordinate $\psi^{\mu}(\tau, \sigma)$. Such a string is called a superstring. One can also have internal degrees of freedom distributed along the string. In this case one has a heterotic string. In addition strings can be both open and closed.

Classically one can find the most general motion of a string and in general the dynamics of a string can be understood as arising from the balance between the centrifugal force that will tend to make the string longer and the force due to the string tension that will tend to push a string to become a point.

When a string is quantized one finds an infinite spectrum of states lying on linearly rising Regge trajectories with slope $\alpha^{\prime}$ related to the string tension through the relation $T=\left[2 \pi \alpha^{\prime}\right]^{-1}$. In particular the spectrum contains a massless sector with spin 1 gauge fields and a spin 2 graviton showing that the theory is able to unify gauge interactions with Einstein's theory of general relativity. In addition in the case of superstring and heterotic string, that are the only theories free from infrared problems as for instance tachyons and therefore the only fully consistent ones, one finds that they are supersymmetric. It is actually this symmetry that saves them from the inconsistencies of the bosonic string. Therefore the existence of a new symmetry between bosons and fermions, called supersymmetry, is a prediction of string theory.

A string theory has a huge spectrum of massive states with masses and degeneracy given respectively by $M^{2}=N / \alpha^{\prime}$ and by $d(M) \equiv e^{c \sqrt{\alpha^{\prime}} M}$, where $c$ is a number depending on the particular string theory and $N$ is a non negative integer.

In the field theory limit corresponding to the infinite tension limit $(T \rightarrow \infty$, $\alpha^{\prime} \rightarrow 0$ ), that is the limit where a string becomes a particle because, in this limit, 
the centrifugal force is not able anymore to compensate the string tension, we see that only the massless states survive in the spectrum, while all the massive states acquire an infinite mass and disappear from it.

Strings can also interact, but, unlike pointlike particles where the interaction is obtained by introducing interaction vertices and the interaction point is a point of singularity, no interaction vertex is needed for strings and the world tubes of strings join smoothly.

It turns out that superstrings and heterotic strings cannot be consistently quantized unless the space-time dimension of Minkowski space $D \leq 10$. In particular the supersymmetric strings live naturally at $D=10$ where one finds five fully consistent and inequivalent string theories. They are all supersymmetric, four of them are theories of closed strings, while one is a theory of both open and closed strings. Two of them do not include gauge interactions, while the other three include them but with only two very special gauge groups: either $S O(32)$ or $E_{8} \otimes E_{8}$. In order to make contact with the observed phenomenology at presently available energies one must assume that 6 of the 10 dimensions are not expanding as the other 4 . They remained small, highly curved and compactified à la Kaluza-Klein.

Concerning the unification of the gauge couplings, string theory is more powerful than field theory because now also gravity is fully unified with the gauge interactions. In particular, in the heterotic string at tree level at the unification scale one finds [5] a relation between the Newton's constant $G_{N}$, the string coupling constant $g_{s}$ and the gauge coupling constants of the various gauge groups $g_{i}$ 's given by:

$$
\frac{8 \pi G_{N}}{\alpha^{\prime}}=g_{i}^{2} k_{i}=g_{s}^{2}
$$

where the constants $k_{i}$ are the central charges of the Kac-Moody algebras. When one takes into account the one loop corrections one can see that the gauge coupling constants will run according to the renormalization group and the scale of the grand unification can be computed in terms of the string coupling constant and the Planck mass obtaining [6] ( $\gamma$ is the Euler-Mascheroni constant):

$$
M_{G U T}=\frac{e^{(1-\gamma) / 2} 3^{-3 / 4}}{4 \pi} g_{s} M_{P}=5.27 \cdot 10^{17} g_{s} G e V
$$

But, since $g_{s} \sim 1$, as follows from the second equation in eq. (1.2) where $g_{s}$ must be identified with $g_{G U T}$, one gets a discrepancy of a factor $20 \sim 30$ between eq. (2.3) and the first equation in eq. (1.2). This discrepancy is independent on the specific compactification of the six extra dimensions and goes under the name of the string gauge coupling unification problem. In conclusion, although one apriori could have expected that the unification scale of the gauge and gravitational interactions should have been of the order of the Planck mass, it turns out that such a scale is clearly smaller than $M_{P}$ as follows from eq. (2.3), but, however, still a factor $20 \sim 30$ too high in comparison with the extrapolation from the low energy experiments. The various possible resolutions of this problem are reviewed in Ref. [7]. 
We have seen that, in order to make contact with the observed phenomenology, one must compactify six of the ten dimensions. They can be compactified in various ways obtaining a huge number of four-dimensional consistent string theories. Such huge arbitrariness seemed in contradiction with the uniqueness character that a unified theory of all forces is supposed to possess. Although we now understand that the huge amount of arbitrariness is just a consequence of the many possible ways in which we can Kaluza-Klein compactify the ten-dimensional consistent theories, it is, however, still unpleasant to have to accept the existence in ten dimensions of five consistent and inequivalent theories. Why five and not just one if this is the fundamental theory of all interactions?

\section{Non-perturbative equivalence and $M$-theory}

In order to be able to answer the previous question that has puzzled string theorists for more than ten years, we must step back for a second, go back to field theory and see if it is conceivable there that two theories, that are very different when we study them order by order in their respective perturbation theories, can be actually completely equivalent if we analyze them with non-perturbative methods. Actually it turns out that this possibility is not only abstractly open in field theory, but is also realized in some systems in two dimensions as in the Ising model or in the case of the quantum equivalence between the sine-Gordon and Thirring theory [8].

If we consider the sine-Gordon theory described by the two-dimensional Lagrangian:

$$
L_{\text {Sine-Gordon }}=\frac{1}{2}\left(\partial_{\mu} \Phi\right)^{2}-\frac{M^{2}}{\beta} \cos \beta \Phi
$$

and the Thirring theory described by

$$
L_{\text {Thirring }}=\bar{\Psi}\left(\gamma^{\mu} \partial_{\mu}-m\right) \Psi+\frac{g}{2}\left(\bar{\Psi} \gamma^{\mu} \Psi\right)^{2}
$$

it has been shown [8] that, although they look very different and one is a bosonic theory while the other is a fermionic one, they are actually equivalent provided that the two coupling constants are related through the relation:

$$
\beta^{2}=\frac{4 \pi}{1+g / \pi}
$$

As a consequence strong coupling in the Thirring model implies weak coupling in the sine-Gordon theory and viceversa. It can also be seen that the sine-Gordon theory has soliton solutions of the classical equations of motion corresponding in the quantum theory to particles with a mass proportional to the square of the inverse of the sine-Gordon coupling constant $\beta$. They are not appearing as fields in the sine-Gordon Lagrangian, but are in fact described in the Thirring theory by the fundamental field $\Psi$. 
Another example is the two-dimensional Ising model where the partition function $Z(K)$ computed on a square two-dimensional lattice

$$
Z(K) \equiv \sum_{\sigma= \pm 1} e^{K \sum_{<i j>} \sigma_{i} \sigma_{j}} \quad ; \quad K=\frac{J}{K_{B} T}
$$

turns out to be equal to the one computed on the dual lattice obtained from the original square lattice by taking the points situated at the center of the squares of the lattice:

$$
Z(K)=Z^{*}\left(K^{*}\right) \quad \text { if } \quad \sinh \left(2 K^{*}\right)=\frac{1}{\sinh (2 K)}
$$

where $K_{B}$ is the Boltzmann constant, $T$ is the temperature and $J$ is the coupling constant between two next neighbouring lattice points. The partition function $Z(K)$ gives a good perturbative description of the high-temperature phase, while the partition function computed on the dual lattice $Z^{*}\left(K^{*}\right)$ gives a good perturbative description of the low temperature phase.

From what we have seen up to now one may suspect that the equivalence between totally different theories may only be a two-dimensional phenomenon. This is actually not true because there is by now a overwhelming evidence [9] that this also happens in the case of the four-dimensional supersymmetric Yang-Mills theory with gauge group $S U(2)$ and with four supersymmetric conserved charges, the so-called $N=4$ supersymmetric Yang-Mills theory. In general in this theory the original gauge symmetry is broken by the vacuum expectation value of some scalar fields in such a way that the gauge field corresponding to the unbroken $U(1)$ is left massless as the photon in the standard model, while all the other gauge fields, that we denote $W^{ \pm}$, in analogy with the $W$-boson of the standard model, become massive. This theory has also soliton solutions that are monopoles and dyons with respect to the unbroken $U(1)$. It turns out that this theory with gauge coupling constant $g$ and with the particle content that we have described above is exactly quantum equivalent to a supersymmetric $N=4$ Yang-Mills theory with gauge coupling constant $\hbar / g$ where the fundamental fields are now the ones corresponding to the monopoles together with the dual, in the sense of the electromagnetic duality, of the massless $U(1)$ gauge fields realizing the beautiful Montonen-Olive duality idea [10]. In this last theory the particles corresponding to the massive fields $W^{ \pm}$appear instead as soliton solutions.

In conclusion the message that we get from field theory is that two theories can appear to be very different from each other as far as their classical Lagrangians or their respective weak coupling perturbation theories concern, but they can turn out to be completely quantum equivalent if we include also the non-perturbative effects. They are said to be dual to each other under weak-strong coupling duality that acts on the gauge coupling constant as $g \rightarrow \hbar / g$. Notice that the existence of the Planck constant is essential to match the dimension of the two sides of the previous equation. In particular we see that the fundamental particles that are those 
whose fields are present in the Lagrangian of a theory appear as soliton solutions in the dual theory.

These ideas brought several people to investigate the soliton solutions in various string theories or more precisely in the low energy limit of them corresponding to the various supergravity theories. Through this investigation one has found classical "soliton" solutions corresponding to $p$-dimensional objects called $p$-branes, where $p=0$ for a point particle, $p=1$ for a string and so on, of various types ?. They are

1. Smooth $p$-dimensional solitons with a mass per unit $p$-volume proportional to the inverse of the square of the string coupling constant $g_{s}^{2}$. Those are the generalization to a string theory of the solitons found in field theory

2. New types of $p$-dimensional objects, that are called $D$-branes, having a mass per unit of $p$-volume proportional to the inverse of the string coupling constant $g_{s}$. Those are new types of extended objects that are not present in a field theory.

3. Extended $p$-dimensional objects corresponding to singular black holes.

Thus we find that, although we started from a string theory that by definition includes only one-dimensional objects, the theory contains also other kinds of extended objects that, however, become infinitely heavy when $g_{s} \rightarrow 0$ disappearing from the spectrum. Our string theory is therefore a pure string theory only in the perturbative regime $\left(g_{s} \rightarrow 0\right)$ !

We could ask ourselves if, in analogy with what we have learnt in several examples in field theory, we could expect that our original string theory is equivalent to another theory in which the $p$-branes appear as fundamental states. Is there a duality between a string theory and another string theory or more in general between a string theory and a $p$-brane theory?

In analogy with a particle or a string a $p$-brane is described by its space-time coordinate $x^{\mu}\left(\tau, \sigma_{1}, \sigma_{2}, \ldots, \sigma_{p}\right)$ that is a function of the world-volume coordinates $\sigma_{i}$ of the $p$-brane. But, unlike particles and strings, we are not able to consistently quantize $p$-branes with $p>1$. It is true that a $p$-brane as a string softens the Minkowski space-time divergences, but its world-volume description has now so many degrees of freedom that we have not succeded in constructing a meaningful quantum theory of it.

But do we need to have a consistent quantum theory of an elementary arbitrary $p$-brane? Or in other words does there exist a theory dual to one of the five inequivalent string theories where the fundamental elementary object is a $p$-brane with $p>1$ ?

A detailed analysis [12] shows that there is no real need for it. In fact two of the five ten-dimensional consistent string theories, the type I and the heterotic one both with gauge group $S O(32)$, are dual to each other in the sense of the

${ }^{*}$ For a review of the soliton solutions see Ref. [11]. 
weak-strong duality [13, 14], while a third string theory, the type IIB, is dual to itself, i.e. is self-dual [15]. Finally the other two theories, the type IIA and the heterotic one with gauge group $E_{8} \otimes E_{8}$, are dual to an 11-dimensional particle theory [13, 16]. Actually their perturbative regime $\left(g_{s} \rightarrow 0\right)$ can be obtained from an 11-dimensional theory with the 11-th dimension compactified respectively on a circle of radius $R$ or on a circle with opposite points identified (corresponding to the orbifold $\left.S^{1} / Z_{2}\right)$ in the limit in which the radius $R$, that in the ten-dimensional string metric is proportional to a power $g_{s}^{2 / 3}$ of the string coupling constant, tends to zero. This 11-dimensional theory, called M-theory, has the property of reducing at low energy to a unique 11-dimensional supergravity theory. It has also been shown that it reduces to the other three string theories in suitable limits [16].

In conclusion there is a strong evidence for the existence of a unique theory that in certain specific limits reduces to one of the five consistent string theories in ten dimensions and in these limits it can be given a very precise quantum meaning in perturbation theory in terms of the string coupling constant, but it is otherwise not a pure theory of strings at all.

It has been recently conjectured [17] that the M-theory can be formulated as the $N$ going to infinity limit of the supersymmetric $N \times N$ matrix quantum mechanics describing D0-branes. During the last half a year several checks have confirmed this conjecture. The next few years will tell us if we have finally constructed a useful formulation of a unique theory of all forces.

Before concluding let us mention two very interesting applications of the nonperturbative ideas developed above.

The first concerns the unification of the couplings. In sect. 2 we have discussed a problem concerning the unifications of the couplings in the framework of the perturbative heterotic string. However, from the value of the coupling constant at the unification scale in eq. (1.2) one gets a value of $g_{s} \sim 1$ that is not consistent with the use of perturbation theory. But, if we go away from the perturbative regime, as we have discussed above, one begins to see the 11th dimension and as a consequence the physics is approximately five-dimensional already below the unification scale [18]. In the $E_{8} \otimes E_{8}$ heterotic string the gauge fields live each on one of the two ten-dimensional walls of the 11-dimensional world, while gravity lives in the entire 11-dimensional world. This means that, below the unification scale where the world is approximately five-dimensional, the dimensionless Planck constant is $G_{N} E^{3}$ and not $G_{N} E^{2}$, as in four dimensions, while the gauge coupling constants still run according to a four-dimensional world. This makes the Newton's constant run faster than before and consequently meet the gauge couplings at a lower energy than before. It has been shown in Ref. [18] that this mechanism can cure the problem encountered in the perturbative heterotic string.

The second concerns the calculation of the entropy of a black hole. It is known, since long time, that black holes obey laws directly analogous to the law of thermodynamics. In particular, they have a temperature and an entropy given by $S=A /\left(4 \hbar c G_{N}\right)$, where $A$ is the area of the horizon. This implies that in a statisti- 
cal mechanics description of a black hole one must identify the microstates that are responsible for the entropy. It has been recently possible to identify them [19] in the black holes appearing in string theories and to show that their number correctly reproduces the entropy given by the Bekenstein-Hawking formula in terms of the area of the horizon.

To conclude, as few years before the fall of the Berlin wall it seemed impossible to believe that this event would have been possible in the next few years to come, so few years ago it seemed impossible that we could understand in an analytical way so many aspects of non-perturbative field and string theory and we could end up having a strong evidence for the existence and even a conjectured formulation of a unique theory unifying all forces observed in nature.

On the other hand, as the fall of the Berlin wall did not solve all the problems of the world, so we have a long way to go for understanding this unique theory and especially to see if it will be able to predict what is observed in the high energy experiments where smaller and smaller structures are investigated.

\section{References}

[1] L.D. Landau and E.M. Lifshitz, "The classical theory of fields", AddisonWesley Publishing Company, p. 97.

[2] U. Amaldi, W. de Boer and H. Fürstenau, Phys. Lett. 260B (1991) 447.

[3] J. Scherk and J. Schwarz, Nucl. Phys. B81 (1974) 118.

[4] M. Green, J. Schwarz and E. Witten, "Superstring theory", Cambridge University Press (1987).

[5] P. Ginsparg, Phys. Lett. 197B (1987) 153.

[6] V.S. Kaplunovsky, Nucl. Phys. B307 (1988) 145; Erratum: ibid. B382 (1992) 436.

[7] K.R. Dienes, Nucl. Phys. Proc. Suppl. 52A (1997) 276.

[8] A. Luther and I. Peschel, Phys. Rev. B9 (1974) 2911; S. Coleman, Phys. Rev. D11 (1975) 2088; S. Mandelstam, Phys. Rev. D11 (1975) 3026.

[9] H. Osborn, Phys. Lett. 83B (1979) 321; A. Sen, Phys. Lett 329B (1994) 217; D.I. Olive, "Exact electromagnetic duality", hep/th 9508089.

[10] C. Montonen and D. Olive, Phys. Lett. 72B (1977) 117.

[11] M.J. Duff, Ramzi R. Khuri and J.X. Lu, Phys. Rep. 259 (1995) 326.

[12] C.M. Hull, Nucl. Phys. B468 (1996) 113. 
[13] E. Witten, Nucl. Phys. B443 (1995) 85.

[14] A. Dabholkar, Phys. Lett 357B (1995) 307; C.M. Hull, Phys. Lett 357B (1995) 545; J. Polchinsky and E. Witten, Nucl. Phys. B460 (1996) 525.

[15] C.M. Hull and P. Townsend, Nucl. Phys. B438 (1995) 109.

[16] P. Horava and E. Witten, Nucl. Phys. B460 (1996) 506.

[17] T. Banks, W. Fischler, S.H. Shenker and L. Susskind, Phys. Rev. D55 (1997) 5112.

[18] E. Witten, Nucl. Phys. B471 (1996) 135.

[19] C. Vafa and A. Strominger, Phys. Lett. 379B (1996) 99. 